\title{
Diagnostic Value of Leukocyte Count Abnormalities in Newly Diagnosed Tuberculosis Patients
}

\author{
Bertrand Hugo Mbatchou Ngahane ${ }^{1,2^{*}}$, Abinkeng Tazifua Ebenezer2, \\ Ngouadjeu Dongho Eveline ${ }^{1,2}$, Mafo Nzonteu Estelle Raïssa1, Kemta Lekpa Fernando1, \\ Che Vanessa Lum², Namme Luma Henry ${ }^{1,3}$ \\ ${ }^{1}$ Douala General Hospital, Department of Internal Medicine, Douala, Cameroon \\ ${ }^{2}$ Faculty of Medicine and Pharmaceutical Sciences, University of Douala, Douala, Cameroon \\ ${ }^{3}$ Faculty of Medicine and Biomedical Sciences, University of Yaounde 1, Yaounde, Cameroon \\ Email: *mbatchou.ngahane@yahoo.com
}

How to cite this paper: Mbatchou Ngahane, B.H., Ebenezer, A.T., Eveline, N.D., Raïssa, M.N.E., Fernando, K.L., Lum, C.V. and Henry, N.L. (2020) Diagnostic Value of Leukocyte Count Abnormalities in Newly Diagnosed Tuberculosis Patients. Open Journal of Respiratory Diseases, 10, 1-10.

https://doi.org/10.4236/ojrd.2020.101001

Received: October 14, 2019

Accepted: December 21, 2019

Published: December 24, 2019

Copyright $\odot 2020$ by author(s) and Scientific Research Publishing Inc. This work is licensed under the Creative Commons Attribution International License (CC BY 4.0).

http://creativecommons.org/licenses/by/4.0/

(c) (i) Open Access

\begin{abstract}
Background: The diagnosis of tuberculosis (TB) is frequently challenging given that the clinical and radiographic features of TB are often nonspecific. Altered leukocyte count ratios could serve as new tools of diagnostic orientation of tuberculosis. The aim of this study was to assess the diagnostic value of the leukocyte count ratios for the diagnosis of TB. Methods: This was a cross-sectional study including cases of newly diagnosed TB patients from registers of the TB treatment center of the Douala General Hospital. Control subjects were healthy volunteers, age and sex matched, recruited at the blood bank. Sociodemographic, clinical data and peripheral blood parameters were collected. The diagnostic value of leukocyte counts was determined using receiver operating characteristics curve analysis. Results: In total, $204 \mathrm{~TB}$ patients and 204 control subjects were included in the study. The gender of the participants was equitably distributed in the 2 study groups (male $61.8 \%$; female $38.2 \%$ ). The median age of TB patients was 33 years while that of control patients was 32 years. The monocyte-lymphocyte count ratio (MLR) and neutrophil-lymphocyte count ratio (NLR) were significantly higher in the TB patients group compared to control group. A NLR $>1.19$ and MLR $>0.29$ were identified as cut-off values for discriminating TB patients. The areas under the curves (AUC) were 0.77 and 0.84 for the MLR and NLR respectively. Conclusion: A raised NLR $>1.19$ and MLR $>0.29$ are predictive of tuberculosis. The NLR has greater diagnostic ability as evidenced by its higher AUC. Further research is needed to confirm or refute our findings.
\end{abstract}




\section{Keywords}

Leukocytes, Diagnosis, Tuberculosis, Africa

\section{Introduction}

Tuberculosis (TB) is highly prevalent chronic infectious disease caused by Mycobacterium tuberculosis (MTB), an aerobic intracellular bacterium. MTB is the second leading cause of death from a single infectious agent, after the human immunodeficiency virus (HIV). It ranks amongst the first worldwide pathogens latently infecting one third of the population [1]. This reservoir of infected individuals resulted in an estimated 10.4 million incident TB cases, with 1.4 million deaths reported worldwide in 2015 [1]. In 2014 the African Region had the most severe burden relative to population of the disease with 275 incident cases per 100,000 population on average, more than double the global average of 133 [2]. Cameroon records an estimated 25,000 new cases of TB annually since 2006 while the mortality rate in the general population is $57 / 100,000$ inhabitants and 6\% among notified TB case [1] [3].

For decades, myeloid-specific cells have been known to serve as host cells for MTB growth and lymphoid cells are thought to be the major effector cells in tuberculosis immunity. Given the central role of monocytes and lymphocytes in the induction of immune responses, their levels in peripheral blood might be expected to reflect the state of an individual's immunity to infection. Therefore, the relative abundance may reflect a balance between effector and target cells [4]. Recent clinical analysis of peripheral blood mononuclear cells supports the hypothesis that a significantly high or low monocytes/lymphocytes count ratio (MLR) does not only correlate with risk of developing tuberculosis but is also predictive of active TB [5] [6].

Evidence has emerged in recent years suggesting that neutrophils may also play a role in early innate immunity against MTB. Neutrophils are the most commonly infected phagocytic cell in sputum samples in the airways of patients with active tuberculosis (TB). They may play an important role as part of the innate host response to mycobacterium and contribute to the early control of MTB infection [5] [7]. Previous reports have shown that the neutrophils/lymphocytes count ratio (NLR) is significantly lower in tuberculosis compared to bacterial community acquired pneumonia and sarcoidosis with cut-off values of 7 and 2.5 respectively [8] [9].

Diagnosis of TB is frequently challenging, given that clinical and radiographic features of TB are not specific and acid-fast bacilli sputum smear-the most widely available in resource-limited settings is of a variable sensitivity from $74 \%$ to $93 \%$ and a specificity from $71 \%$ to $96 \%$ [10] [11] [12]. Delays in diagnosing pulmonary tuberculosis can have a negative effect on patient morbidity and mortality and increase disease transmission [9]. Studies on changes in leukocyte 
subsets in newly diagnosed TB patients are discrepant. In addition, values of the NLR and MLR between TB patients and healthy subjects have not been established.

The aim of this study was to find out the association of various leukocyte count ratios, monocytes/lymphocytes, neutrophils/lymphocytes ratios and tuberculosis and to evaluate their diagnostic abilities.

\section{Methods}

\subsection{Study Design and Setting}

A cross-sectional study was conducted in the TB treatment center, and in the blood bank and hematology unit of the laboratory of the DGH from January 2016 to May 2016. This hospital with a capacity of 300 beds is a tertiary care and teaching hospital. In the TB treatment center, all patients diagnosed with $\mathrm{TB}$ are registered in the TB register before starting the treatment. The diagnosis and the treatment are based on guidelines of the national tuberculosis control program described below. The blood bank is part of the diagnostic laboratory of the DGH. The hematology unit is endowed with an automated hematology analyzer and is headed by a clinical hematologist. Socioeconomic and clinical data of donors are collected before blood donation. They also benefit from free HIV and hepatitis B and C screening. Donors are composed mainly of young adults from the Douala sub region as well as family members of hospitalized patients. They are usually healthy, without any clinical signs or symptoms.

\subsection{Participants}

Our study population was made of 2 groups: 1) patients aged 18 years and above, diagnosed TB and registered in the TB treatment center. These included smear positive and negative pulmonary $\mathrm{TB}$, as well as pleural and lymph node $\mathrm{TB}$. Smear negative pulmonary $\mathrm{TB}$ was defined according to the guidelines of the National Tuberculosis Control Programme (13) 2) healthy controls were patients recruited at the blood bank of the DGH. They were age and sex-matched to $\mathrm{TB}$ patients.

Subjects with known chronic conditions such as HIV infection, viral hepatitis, hematological disorders, diabetes mellitus, pregnancy, steroid therapy within 3 months before the study were excluded from both groups. Participants with clinical suspicion of infectious diseases other than tuberculosis were also excluded, as well as patients who did not perform differential white blood cell count.

A consecutive sampling was used for the 2 groups of subjects. TB patients were recruited from TB register while control subjects were consecutively included among blood donors at the blood bank of the DGH.

\subsection{Data Collection}

All the donors provided written informed consent and the study was approved by the ethics committee of the Faculty of Medicine and Pharmaceutical Sciences 
of the University of Douala while administrative clearance was obtained from the Douala General Hospital authorities. Data were collected on sociodemographiccharacteristics such as age, sex and residence; Clinical forms of tuberculosis as well as peripheral blood parameters such as hemoglobin concentration, neutrophils, lymphocytes, monocytes, and platelet counts. About $3-4 \mathrm{ml}$ of peripheral venous blood was drawn aseptically in the morning, with sterile needles and collected into ethylene-diamine tetra-acetic acid containing (EDTA) tubes. Quantitative analysis of blood cells was performed by the automated Cell Dyn Ruby Hematology analyzer (ABBOT model) at the clinical diagnostic laboratory of the Douala General Hospital. The NLR and MLR were evaluated as follows: NLR $=$ absolute neutrophil count/absolute lymphocyte count, MLR $=$ absolute monocyte count/absolute lymphocyte count

The diagnosis of TB was based on the guidelines of the National Tuberculosis Control Programme [13]. Smear-positive pulmonary TB was diagnosed on the basis of clinical symptoms, and the presence of at least one acid fast bacillus $(\mathrm{AFB}+)$ in at least one sputum sample from two samples submitted on 2 consecutive days for microscopic examination after staining by Ziehl-Neelsen's technique. The diagnosis of smear-negative TB was based on no improvement of symptoms after a 10-day course of nonspecific antibiotic therapy, persistent negative results of a new series of two sputum smear examinations, and chest $\mathrm{X}$-ray abnormalities consistent with active pulmonary TB. The diagnosis of extrapulmonary TB was based on suggestive clinical signs and evidence of a predominantly lymphocytic exudate as in the case of pleural or peritoneal TB with ascites or granulomatous lesions on histopathological examination of lymph nodes or other tissue biopsy. Patients with pulmonary TB associated or not with extra pulmonary sites were considered pulmonary cases and recorded as such [13].

\subsection{Data Analysis}

The data were collected and analyzed with the program the IBM Statistical Package for the Social Sciences, version 20.0 (IBM Corp, Armonk, NY, USA). Quantitative data was summarized using the median and interquartile range (IQR). Categorical data were summarized using number and percentage. Comparison of continuous data was done using Mann-whitney test. Chi 2 test was used for the comparison of categorical data. The diagnostic value of leukocyte count was estimated using receiver operating characteristics curve (ROC) analysis for the MLR and NLR. Sensitivity, specificity, negative predictive value (NPV) and positive predictive value (PPV) were also calculated.

The cut-off values of these ratios correspond to the plots on the respective curves that yield the best sensitivity and specificity. The areas under the curves (AUC), which quantifies the ability of the test to differentiate between two outcomes were determined and graded as follow: perfect test (AUC of 1); excellent test (AUC 0.9 - 0.99), good test (AUC 0.8 - 0.89), fair test (AUC $0.7-0.79$ ), non useful test $(\mathrm{AUC}<0.70)$. A P-value less than 0.05 was considered significant. 


\section{Results}

From the 438 files of TB patients selected, we excluded 234 for various reasons as illustrated in Figure 1.

\subsection{General Characteristics of the Study Population}

A total of 204 consecutive diagnosed TB patients' files and 204 blood donors were included in the study. They were $126(61.8 \%)$ males for the two groups giving a male: female sex ratio of 1.61. The median age was 33 (IQR 26 - 42) years and 32 (IQR 25 - 40) years respectively for TB patients and control subjects. As shown in Table 1, pulmonary TB was observed in 95 (46.6\%). There were statistically significant differences concerning total white blood cells and differential counts in the two groups.

Table 1. Baseline characteristics of the participants.

\begin{tabular}{|c|c|c|c|}
\hline Characteristics & $\begin{array}{l}\text { TB patients } \\
(\mathrm{N}=204)\end{array}$ & $\begin{array}{l}\text { Healthy controls } \\
\qquad(\mathrm{N}=204)\end{array}$ & P-value \\
\hline \multicolumn{4}{|l|}{ Sex } \\
\hline Male & $126(61.8 \%)$ & $126(61.8 \%)$ & 0.9 \\
\hline Female & $78(38.2 \%)$ & $78(38.2 \%)$ & \\
\hline \multicolumn{4}{|l|}{ Age } \\
\hline Median age (IQR) & $33.00(26-42)$ & $32.00(25-40)$ & 0.9 \\
\hline$<25$ & $41(20.1 \%)$ & $51(25 \%)$ & \\
\hline $25-34$ & $72(35.3 \%)$ & $68(33.3 \%)$ & \\
\hline $35-44$ & $3(26.0 \%)$ & $51(25 \%)$ & \\
\hline $45-54$ & $29(14.2 \%)$ & $30(14.7 \%)$ & \\
\hline$\geq 55$ & $9(4.4 \%)$ & $4(2.0 \%)$ & \\
\hline \multicolumn{4}{|l|}{ Level of education } \\
\hline Primary school & $53(26 \%)$ & $71(34.8 \%)$ & \\
\hline Secondary school & $91(44.6 \%)$ & $79(38.7 \%)$ & 0.18 \\
\hline University & $60(29.4 \%)$ & $54(26.5 \%)$ & \\
\hline \multicolumn{4}{|l|}{ Tuberculosis } \\
\hline Smear positive pulmonary $\mathrm{TB}$ & $95(46.6 \%)$ & & \\
\hline Smear negative pulmonary TB & $38(18.6 \%)$ & & \\
\hline Extrapulmonary TB & $71(34.8 \%)$ & & \\
\hline \multicolumn{4}{|l|}{ Cell count (Giga/l) (median, IQR) } \\
\hline Total white blood cells & $6.5(4.80-8.47)$ & $4.25(3.68-5.05)$ & $<0.001$ \\
\hline Neutrophils & $4.25(2.83-5.75)$ & $1.87(1.51-2.39)$ & $<0.001$ \\
\hline Lymphocytes & $1.54(1.18-1.96)$ & $1.67(1.42-2.23)$ & 0.002 \\
\hline Monocytes & $0.60(0.38-0.77)$ & $0.32(0.24-0.41)$ & $<0.001$ \\
\hline
\end{tabular}




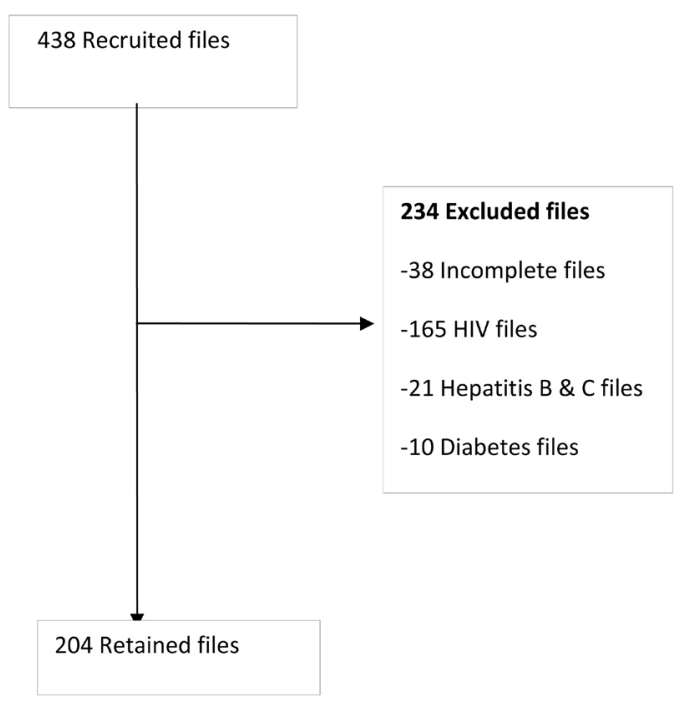

Figure 1. Flow chart.

\subsection{Leukocyte Count Ratios}

The monocyte-lymphocyte count ratio was higher in the patient group (median 0.38 ; IQR $0.24-0.56$ ) as compared to the control group (0.19; IQR $0.14-0.25$ ) $(\mathrm{p}<0.001)$. The neutrophil-lymphocyte count ratio was also significantly increased in the patient group, 2.71 (IQR: $1.62-4.29$ ) in comparison to the control group, 1.03 (IQR: $0.78-1.48)(\mathrm{p}<0.001)$.

\subsection{Neutrophils-Lymphocytes Ratio (NLR) and Monocytes-Lymphocytes Ratio (MLR)}

As illustrated in Figure 2, the area under the curve for NLR (AUC: 0.84) was significantly greater than that for MLR (AUC: 0.76$)(\mathrm{P}<0.001)$. A MLR $>0.29$ was identified as the optimal cut-off value for discriminating patients with $\mathrm{TB}$ from healthy subjects. Using this cut-off value, the MLR showed $67.2 \%$ sensitivity, $83.3 \%$ specificity, a positive predictive value (PPV) of $80.12 \%$ and a negative predictive value of (NPV) of $71.73 \%$. A NLR $>1.79$ was identified as the optimal cut-off value for discriminating patients with TB from healthy subjects, yielding $70.6 \%$ sensitivity, $87.3 \%$ specificity, a PPV of $84.7 \%$ and NPV of $74.79 \%$.

\section{Discussion}

The aim of this study was to determine the relationship between various leukocyte count ratios and TB and to evaluate their diagnostic abilities. The NLR and MLR were significantly raised in TB patients compared to the control group. A MLR $>0.29$ and a NLR $>1.79$ were optimal cut-off values for differentiating TB patients from healthy subjects. The NLR had greater diagnostic ability as evidenced by its high AUC.

The neutrophil lymphocyte count ratio is an expedient marker of inflammation for foreseeing bacterial infection [9] [14]. The physiological immune responses of circulating leukocytes to various stressful events are characterized by 


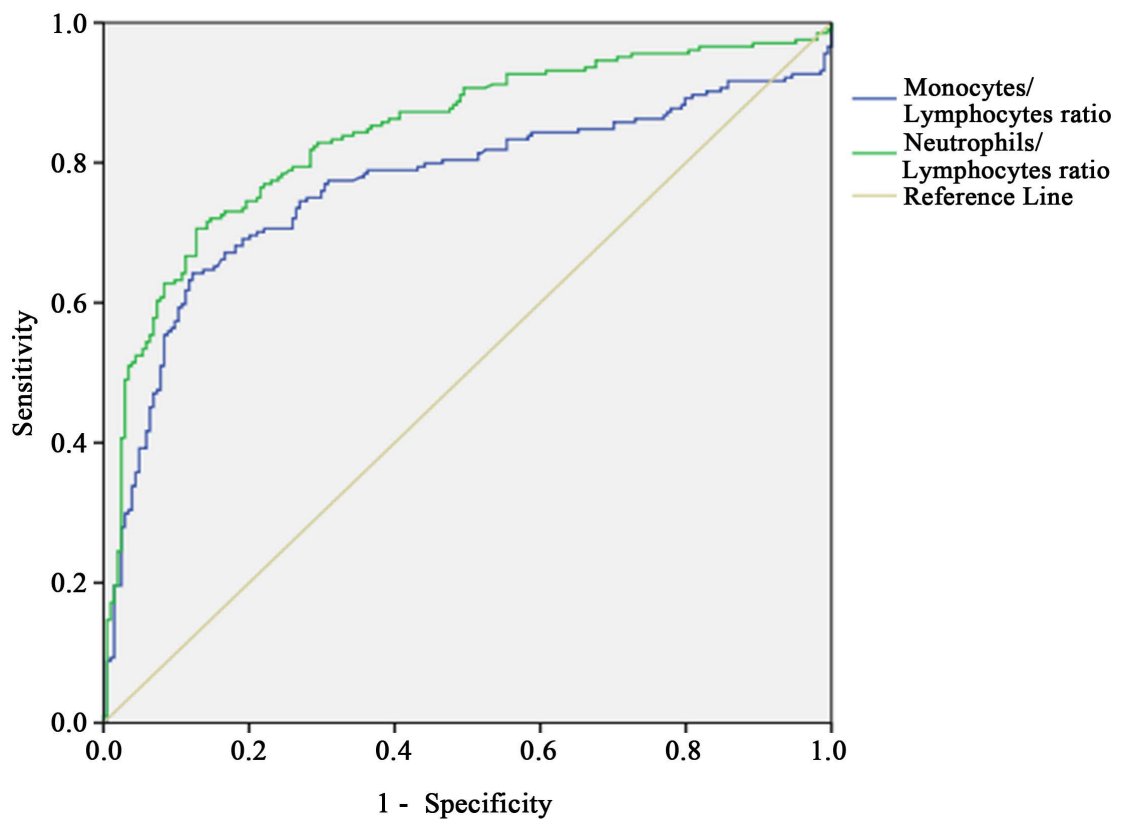

Figure 2. Receiver Operating characteristics curves of MLR and NLR for discriminating TB patients from healthy subjects. 1-specificity (X-axis); Sensitivity (Y axis).

an increased neutrophils count and decreased lymphocyte count. An increase in total WBC and neutrophils is an inflammatory reaction, particularly when caused by a bacterial infection [9]. Therefore, the NLR is thought to have stronger discriminative power for predicting bacteremia compared to discrimination based on neutrophilia or lymphocytopenia alone. Goodman et al. [15], Zahorec et al. [16] and recently, de Jager et al. [17] all demonstrated that the NLR was a better predictor of bacteremia than routine parameters such as CRP level, WBC count, and neutrophil count. Currently, the NLR has been garnering interest as a survival predictor in various clinical situations, ranging from oncological to cardiovascular diseases [18] [19]. Sumaria et al. reported increased NLR (3.12) in newly diagnosed TB patients compared to healthy controls (1.92) [14]. These observations were similar to our findings of 2.73 for the TB group and 1.03 for controls.

Monocytes are an essential component of the innate immune response that acts as a link to the adaptive immune system through antigen presentation to lymphocytes. Thus any factor that perturbs the function or relative numbers of either cell type could potentially affect an individual's response to infection [4]. The normal ML ratio is disrupted by MTB infection. It has been reported recently that MTB infection may alter subsets of hematopoietic stem cells [20] or directly infect bone marrow mesenchymal stem cells [21]. Studies in mice and humans have shown that subsets of hematopoietic stem cells have distinct biases in the ratio of myeloid to lymphoid cells they give rise to [22] [23] [24] [25]. The different proportion of myeloid biased or lymphoid-biased hematopoietic stem cells may underlie the peripheral difference of ML ratio. Therefore, it is reasonable that MTB infection may alter hematopoietic stem cells such that the ML ratio 
is altered. The change of monocytes and lymphocytes, reflected in their ratio, may affect patients' ability to respond to mycobacterial infection. As demonstrated by our study, patients with active tuberculosis had a higher ML ratio compared to healthy controls, coinciding with the results of Jun Wang et al. 2015 [4].

Yoon 2013 identified a NLR $<7$ as an optimal cut-off for differentiating pulmonary TB from bacterial community-acquired pneumonia [9]. Iliaz 2014 found a NLR $<2.5$ as cut-off for differentiating TB from sarcoidosis [8]. We found a NLR $<1.19$ and a MLR $<0.29$ as cut-off for discriminating healthy subjects from TB patients. The NLR (AUC: 0.84) was a more powerful discriminative marker than the MLR (AUC: 0.77) as evidenced by its higher AUC. The NLR is thus a good while the MLR is a fair diagnostic test.

Although the present study is among the first studies to demonstrate and compare the diagnostic abilities of the neutrophil-lmphocyte and monocytelymphocyte count ratios in discriminating TB patients from healthy subjects, it faces some limitations. Firstly, it was a single center study, limitating the generalisability of our results. Also, the control group was not screened biologically for $\mathrm{TB}$, raising the possibility of inclusion of subjects with subclinical disease among the controls. Furthermore, diagnosis of some forms of TB in this study was considered on the basis of suggestive clinical signs and response to treatment. This may be another source of bias in this study.

\section{Conclusion}

The neutrophil and monocyte-lymphocyte count ratios are raised in active TB. A NLR $>1.19$ and MLR $>0.29$ are optimal cut-offs to differentiate healthy subjects from TB patients. The NLR has greater diagnostic ability as evidenced by its higher AUC. Further research is needed to confirm or refute our findings.

\section{Authors' Contributions}

Conception and Design: MNBH, ATE; Data collection: ATE; Data analysis and Interpretation: $\mathrm{MNBH}$; Drafting of the manuscript: ATE. Reviewing the manuscript: MNBH, NDE, ATE, MNER, KLF, CVL, NLH. All authors approved the final draft for publication.

\section{Acknowledgements}

We thank the International Multidisciplinary Programme to Adress Lung Health in Africa (IMPALA) for its support.

\section{Conflicts of Interest}

The authors declare no conflicts of interest.

\section{References}

[1] World Health Organization (2016) Global Tuberculosis Report. Geneva, Switzer- 
land.

[2] Zumla, A., George, A., Sharma, V., Herbert, R.H.N., Baroness Masham of Ilton, Oxley, A., et al. (2015) The WHO 2014 Global Tuberculosis Report-Further to Go. The Lancet Global Health, 3, e10-e12. https://doi.org/10.1016/S2214-109X(14)70361-4

[3] Noeske, J., Nana Yakam, A. and Abena Foe, J.-L. (2016) Epidemiology of Tuberculosis in Cameroon as Mirrored in Notification Data, 2006-2014. The International Journal of Tuberculosis and Lung Disease, 20, 1489-1494. https://doi.org/10.5588/ijtld.16.0252

[4] Wang, J., Yin, Y., Wang, X., Pei, H., Kuai, S., Gu, L., et al. (2015) Ratio of Monocytes to Lymphocytes in Peripheral Blood in Patients Diagnosed with Active Tuberculosis. The Brazilian Journal of Infectious Diseases, 19, 125-131. https://doi.org/10.1016/j.bjid.2014.10.008

[5] Eum, S.-Y., Kong, J.-H., Hong, M.-S., Lee, Y.-J., Kim, J.-H., Hwang, S.-H., et al. (2010) Neutrophils Are the Predominant Infected Phagocytic Cells in the Airways of Patients with Active Pulmonary TB. Chest, 137, 122-128.

https://doi.org/10.1378/chest.09-0903

[6] Naranbhai, V., Hill, A.V.S., Abdool Karim, S.S., Naidoo, K., Abdool Karim, Q., Warimwe, G.M., et al. (2014) Ratio of Monocytes to Lymphocytes in Peripheral Blood Identifies Adults at Risk of Incident Tuberculosis among HIV-Infected Adults Initiating Antiretroviral Therapy. The Journal of Infectious Diseases, 209, 500-509. https://doi.org/10.1093/infdis/jit494

[7] Lowe, D.M., Redford, P.S., Wilkinson, R.J., O’Garra, A. and Martineau, A.R. (2012) Neutrophils in Tuberculosis: Friend or Foe? Trends in Immunology, 33, 14-25. https://doi.org/10.1016/j.it.2011.10.003

[8] Iliaz, S., Iliaz, R., Ortakoylu, G., Bahadir, A., Bagci, B. and Caglar, E. (2014) Value of Neutrophil/Lymphocyte Ratio in the Differential Diagnosis of Sarcoidosis and Tuberculosis. Annals of Thoracic Medicine, 9, 232-235. https://doi.org/10.4103/1817-1737.140135

[9] Yoon, N.-B., Son, C. and Um, S.-J. (2013) Role of the Neutrophil-Lymphocyte Count Ratio in the Differential Diagnosis between Pulmonary Tuberculosis and Bacterial Community-Acquired Pneumonia. Annals of Laboratory Medicine, 33, 105-110. https://doi.org/10.3343/alm.2013.33.2.105

[10] Odubanjo, M.O. and Dada-Adegbola, H.O. (2011) The Microbiological Diagnosis of Tuberculosis in a Resource-Limited Setting: Is Acid-Fast Bacilli Microscopy Alone Sufficient? Annals of Ibadan Postgraduate Medicine, 9, 24-29. https://doi.org/10.4314/aipm.v9i1.72431

[11] Shea, Y.R., Davis, J.L., Huang, L., Kovacs, J.A., Masur, H., Mulindwa, F., et al. (2009) High Sensitivity and Specificity of Acid-Fast Microscopy for Diagnosis of Pulmonary Tuberculosis in an African Population with a High Prevalence of $\mathrm{Hu}-$ man Immunodeficiency Virus. Journal of Clinical Microbiology, 47, 1553-1555. https://doi.org/10.1128/JCM.00348-09

[12] Farnia, P., Mohammadi, F., Zarifi, Z., Tabatabee, D.J., Ganavi, J., Ghazisaeedi, K., et al. (2002) Improving Sensitivity of Direct Microscopy for Detection of Acid-Fast Bacilli in Sputum: Use of Chitin in Mucus Digestion. Journal of Clinical Microbiology, 40, 508-511. https://doi.org/10.1128/JCM.40.2.508-511.2002

[13] Ministère de la santé publique (2012) Programme National de Lutte contre la Tuberculose. Guide technique pour le personnel de santé. Yaoundé, Cameroun.

[14] Iqbal, S., Ahmed, U. and Khan, M.A. (2015) Haematological Parameters Altered in 
Tuberculosis. Pakistan Journal of Physiology, 11, 13-16.

[15] Goodman, D.A., Goodman, C.B. and Monk, J.S. (1995) Use of the Neutrophil: Lymphocyte Ratio in the Diagnosis of Appendicitis. The American Surgeon, 61, 257-259.

[16] Zahorec, R. (2001) Ratio of Neutrophil to Lymphocyte Counts-Rapid and Simple Parameter of Systemic Inflammation and Stress in Critically Ill. Bratislavské lekárske listy, 102, 5-14.

[17] de Jager, C.P., van Wijk, P.T., Mathoera, R.B., de Jongh-Leuvenink, J., van der Poll, T. and Wever, P.C. (2010) Lymphocytopenia and Neutrophil-Lymphocyte Count Ratio Predict Bacteremia Better Than Conventional Infection Markers in an Emergency Care Unit. Critical Care, 14, R192. https://doi.org/10.1186/cc9309

[18] Gibson, P.H., Croal, B.L., Cuthbertson, B.H., Small, G.R., Ifezulike, A.I., Gibson, G., et al. (2007) Preoperative Neutrophil-Lymphocyte Ratio and Outcome from Coronary Artery Bypass Grafting. American Heart Journal, 154, 995-1002. https://doi.org/10.1016/j.ahj.2007.06.043

[19] Sarraf, K.M., Belcher, E., Raevsky, E., Nicholson, A.G., Goldstraw, P. and Lim, E. (2009) Neutrophil/Lymphocyte Ratio and Its Association with Survival after Complete Resection in Non-Small Cell Lung Cancer. The Journal of Thoracic and Cardiovascular Surgery, 137, 425-428. https://doi.org/10.1016/j.jtcvs.2008.05.046

[20] Baldridge, M.T., King, K.Y., Boles, N.C., Weksberg, D.C. and Goodell, M.A. (2010) Quiescent Haematopoietic Stem Cells Are Activated by IFN- $\gamma$ in Response to Chronic Infection. Nature, 465, 793-797. https://doi.org/10.1038/nature09135

[21] Das, B., Kashino, S.S., Pulu, I., Kalita, D., Swami, V., Yeger, H., et al. (2013) CD271+ Bone Marrow Mesenchymal Stem Cells May Provide a Niche for Dormant Mycobacterium Tuberculosis. Science Translational Medicine, 5, 170 ra13. https://doi.org/10.1126/scitranslmed.3004912

[22] Müller-Sieburg, C.E., Cho, R.H., Thoman, M., Adkins, B. and Sieburg, H.B. (2002) Deterministic Regulation of Hematopoietic Stem Cell Self-Renewal and Differentiation. Blood, 100, 1302-1309. https://doi.org/10.1182/blood.V100.4.1302.h81602001302_1302_1309

[23] Pang, W.W., Price, E.A., Sahoo, D., Beerman, I., Maloney, W.J., Rossi, D.J., et al. (2011) Human Bone Marrow Hematopoietic Stem Cells Are Increased in Frequency and Myeloid-Biased with Age. Proceedings of the National Academy of Sciences of the United States of America, 108, 20012-20017. https://doi.org/10.1073/pnas.1116110108

[24] Muller-Sieburg, C.E., Sieburg, H.B., Bernitz, J.M. and Cattarossi, G. (2012) Stem Cell Heterogeneity: Implications for Aging and Regenerative Medicine. Blood, 119, 3900-3907. https://doi.org/10.1182/blood-2011-12-376749

[25] Schroeder, T. (2010) Hematopoietic Stem Cell Heterogeneity: Subtypes, Not Unpredictable Behavior. Cell Stem Cell, 6, 203-207. https://doi.org/10.1016/j.stem.2010.02.006 